SMALL INTESTINE

\title{
Chemical nociception in the jejunum induced by capsaicin
}

\author{
B Schmidt, J Hammer, P Holzer, H F Hammer
}

Gut 2004;53:1109-1116. doi: 10.1136/gut.2003.029793

See end of article for authors' affiliations .....................

Correspondence to: Dr J Hammer Universitätsklinik für Innere Medizin IV, Abteilung für Gastroenterologie und Hepatologie, Währinger Gürtel 18-20, A-1090 Vienna, Austria; Johann.Hammer@ univie.ac.at

Accepted for publication 28 October 2003

\begin{abstract}
Background and aims: Chemonociception in the human small intestine has not been studied extensively. Although capsaicin can cause intestinal sensations, it is not known if this is due to stimulation of chemoreceptors or to motor changes. Our aims were to evaluate motor activity during capsaicin induced nociception and to compare qualities of jejunal nociception induced by capsaicin and mechanical distension.

Methods: Twenty nine healthy subjects swallowed a tube with a perfusion site at the ligament of Treitz and, $7 \mathrm{~cm}$ distally, a barostat balloon. Phasic motor activity was measured around the perfusion site and the balloon. Capsaicin solutions (40,200, and $400 \mu \mathrm{g} / \mathrm{ml}) 2.5 \mathrm{ml} / \mathrm{min}$ were perfused for 60 minutes or until severe discomfort occurred. A graded questionnaire for seven different sensations was completed every 10 minutes and after capsaicin perfusion was replaced by saline perfusion because of severe discomfort. Sensations arising from pressure controlled distensions were assessed before and after capsaicin perfusion when sensations had stopped $(n=19)$, or during capsaicin administration when no discomfort was reported $(n=5)$.

Results: Capsaicin perfusion induced feelings of pressure, cramps, pain, and warmth. The quality and abdominal location of these sensations were similar to those induced by distension, except for warmth $(p<0.01)$ and pressure $(p<0.05)$. Seven of 12 subjects receiving $40 \mu \mathrm{g} / \mathrm{ml}$ capsaicin and all subjects receiving higher capsaicin concentrations developed discomfort. Perfusion had to be stopped after 55 (3.3), 15 (5.7), and 10 (2.2) minutes with 40,200 , and $400 \mu \mathrm{g} / \mathrm{ml}$ capsaicin, respectively, whereafter the sensations disappeared within 10 minutes. Repeated capsaicin $(200 \mu \mathrm{g} / \mathrm{ml})$ applications significantly reduced the time until discomfort occurred $(p=0.01)$. Jejunal tone was not altered by capsaicin but phasic activity proximal to the perfusion site was reduced during capsaicin induced discomfort $(p<0.001)$. Pain thresholds during distensions were not different before and after capsaicin perfusion.

Conclusion: Despite the similarities in abdominal localisation and perceptional quality of capsaicin and distension induced sensations, our results rule out the fact that abdominal discomfort evoked by capsaicin involves sensitisation of mechanoreceptors or an increase in phasic and tonic motor activity. Capsaicin evokes abdominal sensations by stimulation of chemoreceptors which proves the existence of chemonociception in the human small intestine.
\end{abstract}

$\mathrm{V}$ isceral abdominal pain and non-painful sensations are a common experience in health and disease and can either arise from conditions such as inflammation or distension of the gastrointestinal tract or be present without detectable organic disease in so-called functional bowel disorders. ${ }^{1}$ The mechanisms whereby painful and nonpainful sensations are elicited in the gastrointestinal tract are incompletely understood. Clinically, painful and nonpainful sensations are currently thought to arise from abnormal motility patterns such as cramps or distension, ischaemia, or inflammation. ${ }^{2}$

Experimental studies of visceral sensation and pain have mainly used two modes of sensory stimulation-that is, mechanical distension and transmucosal electrical stimulation. Distension is believed to induce sensation by specific stimulation of mechanoreceptors. In healthy subjects, distension results in clinical-type abdominal symptoms that include colicky sensations, stinging or sharp sensations, paraesthesia, or flutter-like sensations, and feeling of abdominal distension or vacuum. ${ }^{3}$ Transmucosal electrical stimulation of the small intestine stimulates afferent pathways non-specifically-that is, without relying on specific receptors. ${ }^{45}$ It results in subjective sensations similar to those elicited by balloon distension. ${ }^{3}$

Studies evaluating visceral sensation and pain using mechanical distension or transmucosal electrical stimulation have demonstrated that patients with functional bowel disorders, such as functional dyspepsia or irritable bowel syndrome, have decreased sensory thresholds for mechanical distension compared with healthy controls. ${ }^{367}$ In contrast, thresholds for electrical intestinal stimulation are similar in healthy subjects and patients with irritable bowel syndrome. ${ }^{3}$ Hypersensitivity for mechanical distension is not confined to a specific region of the gut. Both in functional dyspepsia, where symptoms suggest the involvement of the stomach, and irritable bowel syndrome, where symptoms suggest the involvement of the colon, hypersensitivity affects additional regions of the gastrointestinal tract such as the jejunum ${ }^{3} 8$ and even the oesophagus. ${ }^{9}$ Sensitivity to somatic pain, on the other hand, is normal in functional bowel disorders. ${ }^{37}$

Although chemoreceptors have been shown to be present in the gastrointestinal tract and to be involved in the regulation of various functions, ${ }^{10}$ as well as in the pathogenesis of symptoms, ${ }^{11}$ chemical nociception in the human small intestine has to date not been studied extensively. In a study performed to evaluate the effect of capsaicin on mucosal function in the jejunum, we have recently shown that in fasted healthy volunteers jejunal perfusion with capsaicin causes upper abdominal pain in a concentration and time dependent manner. ${ }^{12}$ Moreover, capsaicin applied topically in an ileostoma similarly induced painful sensations. ${ }^{13}$ This sensation of pain is consistent with the high algesic potency of capsaicin, which is due to its stimulant action on nociceptors. $^{14}$ As previous studies were not primarily 
designed to address the question of pain, ${ }^{12}$ it was not possible to infer whether capsaicin caused pain by directly stimulating nociceptive afferents in the intestinal mucosa or by stimulating motility, ${ }^{15}{ }^{16}$ as might be deduced from the crampy nature of the sensation.

Capsaicin is a well established tool in the study of chemoand thermonociception. ${ }^{14}$ This vanilloid produces its characteristic effects on nociceptive afferent neurones by stimulating specific capsaicin receptors, also termed vanilloid receptors of type $1 .{ }^{17}$ These receptors are cation channels in the cell membrane which serve as transducers for both noxious thermal and chemical stimuli. ${ }^{17}{ }^{18}$ Thus vanilloid type 1 receptor is not only activated by capsaicin and related chemicals ${ }^{19}$ but also, for example, by protons and arachidonyl derivatives such as anandamide $e^{20}$ and 12-(S)-HPETE. ${ }^{21}$

This study was designed to examine whether painful and non-painful perceptions during jejunal perfusion with capsaicin are brought about by alterations in jejunal motility. The specific aims were: firstly, to compare the quality of perceptions during intestinal distension and capsaicin perfusion; secondly, to evaluate whether pretreatment with capsaicin increases perception of intestinal distension; thirdly, to assess whether sensations during capsaicin perfusion are associated with changes in tonic and phasic motility; and fourthly, to assess whether there is sensitisation to repeated capsaicin perfusions.

\section{METHODS \\ Subjects}

Twenty nine healthy volunteers ( 17 females, 12 males; mean age 26 years (range 20-41)) were recruited by public advertisement. None had a history of gastrointestinal complaints or abdominal surgery (except appendectomy and cholecystectomy) and none used medications on a regular basis. All subjects gave written informed consent for the protocol that was approved by the ethics committee of the University Hospital of Vienna, Austria.

\section{Tube assembly}

The 7 lumen orojejunal tube assembly (outer diameter $7 \mathrm{~mm}$ ) was designed to allow perfusion of test solutions, quantification of tonic and phasic jejunal motility, and balloon distension. Four lumina were used for water perfused manometry; manometry ports were positioned $1 \mathrm{~cm}$ (manometry port 4), $6 \mathrm{~cm}$ (port 3), $17 \mathrm{~cm}$ (port 2), and $27 \mathrm{~cm}$ (port 1) from the tip of the tube. The port for perfusion of the test solutions (inner diameter $1 \mathrm{~mm}$ ) opened $22 \mathrm{~cm}$ orad from the tip of the tube between manometry ports 1 and 2 . Between $8 \mathrm{~cm}$ and $15 \mathrm{~cm}$ from the tip of the tube (that is, between manometry port 2 and port 3), a $7 \mathrm{~cm}$ long polyethylene bag was tied firmly to the tube and proximally linked to a computer driven electronic barostat (Dual Drive Barostat, Distender Series II; G\&J Electronics Inc., Toronto, Canada). Two lumina of the tube assembly opened into the barostat balloon. The larger lumen (inner diameter $3 \mathrm{~mm}$ ) was used for insufflation and deflation of the bag and a smaller lumen (inner diameter $1 \mathrm{~mm}$ ) for measuring pressure within the bag. In summary, the perfusion port and two manometry ports were proximal and two manometry ports were distal to the barostat bag. One manometry port was proximal to the perfusion port.

\section{Phasic and tonic motor activity in the jejunum}

The manometry ports were perfused at $0.1 \mathrm{ml} / \mathrm{min}$ with deionised water, using a pneumohydraulic capillary perfusion system. Perfusion tubes were connected to pressure transducers (Oakfield Instruments Ltd., Oxon, UK) and transducer signals were recorded by a computer system for further analysis.
Tonic motor activity was quantified with the barostat device set in a pressure controlled mode. The electronic balloon barostat was originally developed to measure smooth muscle tone in the gastric fundus but has since also been validated in other regions of the gastrointestinal tract. The barostat consists of a nearly infinite compliant polyethylene bag which is simultaneously connected to an inflation reservoir and a pressure transducer, both linked to a computer control system. Once placed in the organ of interest, the balloon is inflated to the operating pressure, which is $2 \mathrm{~mm} \mathrm{Hg}$ above the pressure at which volume changes associated with deep coughing or breathing can be seen. The volume of air injected or withdrawn to maintain the operating pressure is recorded. Changes in the intrabag volume are inversely related to the tone of the organ. Thus an increase in the volume in the bag (at a set intrabag pressure) equals a decrease in organ tone, and vice versa. The barostat balloon lightly distends the intestinal wall but has been shown not to occlude the lumen and to allow passage of intestinal contents. ${ }^{22}$

\section{Random distension protocol}

Jejunal distension was achieved by balloon inflation with eight predetermined pressures from 0 to $42 \mathrm{~mm} \mathrm{Hg}$ above the operating pressure at $6 \mathrm{~mm} \mathrm{Hg}$ increments. Individual stimuli of 90 seconds duration were applied in random order and with interstimulus intervals of 90 seconds, during which the pressure in the barostat bag was returned to the operating pressure. The inflation rate of the barostat balloon was set at $90 \mathrm{ml} / \mathrm{s}$. No distensions were performed during phase III of the interdigestive motor complex and 10 minutes thereafter. Distensions that had been performed within 10 minutes of the onset of a phase III were repeated afterwards. During each distension, subjects completed a perception questionnaire, as described below. If they reported distension induced discomfort for more than 30 seconds (score 5 or higher in questions 1-6) or more than moderate pain (score 3.5 or higher in question 7), the distension stimulus was terminated. Distensions with higher pressures were not performed in these subjects.

\section{Capsaicin solutions}

The jejunum was perfused with an electrolyte solution containing $135 \mathrm{mM}$ sodium, $5 \mathrm{mM}$ potassium, $110 \mathrm{mM}$ chloride, and $30 \mathrm{mM}$ bicarbonate at a rate of $2.5 \mathrm{ml} / \mathrm{min}$. Capsaicin $40 \mu \mathrm{g} / \mathrm{ml}(\mathrm{n}=12), 200 \mu \mathrm{g} / \mathrm{ml}(\mathrm{n}=6)$, or $400 \mu \mathrm{g} / \mathrm{ml}$ $(\mathrm{n}=6)$ were added to the solution. Subjects were randomised to one perfusion concentration and were blinded in terms of which solution they received.

\section{Symptom assessment and anatomical questionnaire}

Before the end of each distension, and every 10 minutes during perfusion with capsaicin, subjects were asked to score the perception they experienced on a graded questionnaire to measure the intensity and type of sensation perceived. The questionnaire included seven graphic rating scales for scoring: (1) sensation of abdominal pressure or fullness; (2) sensation of cramps or colics; (3) stinging or sharp sensation; (4) flutter-like sensation; (5) warmth sensation; (6) sensation of vacuum; and (7) pain. Each rating scale was graded from 0 to 6, except the scale for pain which was graded from 0 to 5 . A score of 0 represented no perception; score 1 represented vague perception of a mild sensation; score 2 definite perception of mild sensation; scores 3 and 4 moderately and highly intense perception of sensation, respectively; score 5 discomfort; and score 6 a painful sensation. The scale assessing pain was graded from 0 (no pain) to 5 (maximal pain). All participants received standard instructions and were told that they could mark half unit 
scores if appropriate. This type of questionnaire has been validated to be reproducible during repeated stimuli, and to show stimulus related perception using graded stimulation. Furthermore, this questionnaire has been validated to demonstrate significant and reproducible changes in perception under different experimental conditions, and differential responses to various stimuli. ${ }^{5}$

At the end of a distension series, as well as at the end of the capsaicin perfusion, an anatomical questionnaire was presented, showing the abdomen divided into nine areas corresponding to the epigastrium, periumbilical area, hypogastrium, both hypochondria, flanks, and ileal fossae. Subjects were asked to mark the locations where the sensations were perceived. Both questionnaires were explained to the subjects in a standardised fashion before the study and during the stepwise distension procedure that was used to familiarise subjects with the distension procedure (see experimental design).

\section{Experimental design}

In the first set of experiments (part $1, \mathrm{n}=24$ ), the effect of capsaicin pretreatment or simultaneous capsaicin administration on mechanosensitivity was tested. Two sets of pressure controlled distensions were performed. The first distension preceded perfusion with capsaicin. The second distension was performed either during or after capsaicin perfusion. In those subjects in whom capsaicin perfusion had to be stopped because of the development of discomfort, the second distension was performed after symptoms had completely disappeared (score $\leqslant 1$ ) (group A). In those subjects who did not develop discomfort during capsaicin perfusion, the second distension was performed after 60 minutes while the capsaicin perfusion continued (group B).

In a second set of experiments (part 2, $n=5$ ), we evaluated whether there is sensitisation to repeated capsaicin perfusions. For this purpose, capsaicin perfusion was stopped when discomfort was reported and resumed after symptoms had disappeared (score $\leqslant 1$ ). Capsaicin perfusion again was stopped when discomfort developed and again resumed afterwards until discomfort reappeared.

Part $1(n=24)$

Participants swallowed the tube assembly after an overnight fast. The tube assembly was positioned under fluoroscopic control in the proximal jejunum so that the perfusion site was located at the ligament of Treitz. After a 10 minute rest period, manometry recording was started and performed throughout the study and balloon distension was performed by a stepwise increase (at $2 \mathrm{~mm} \mathrm{Hg}$ ) of the intraballoon pressure. This distension served to determine the operating pressure and to instruct subjects about the sensations they were expected to report.

Thereafter the barostat bag was deflated to the operating pressure and 10 minutes later the first random distension protocol was performed (distension 1, before capsaicin). After this, perfusion of capsaicin was started during which jejunal phasic activity and jejunal tone were continuously recorded. Every 10 minutes during perfusion and when capsaicin perfusion was stopped, participants completed the symptom questionnaire to assess the sensations that occurred. When participants reported discomfort, perfusion of capsaicin was terminated and the jejunum was perfused with normal saline until the sensations vanished (score $\leqslant 1$ ).

Thereafter the second distension protocol was performed (distension 2, after capsaicin; group A). If participants did not experience discomfort within 60 minutes of jejunal perfusion with capsaicin, perfusion was continued throughout the ensuing second set of randomised distensions (distension 2, during capsaicin; group B). After completion of the second randomised distension protocol, the barostat balloon was deflated and the tube gently removed.

\section{Part $2(n=5)$}

In the second set of experiments, subjects also swallowed an orojejunal tube. Manometric recordings were performed only to document the absence of a phase III interdigestive motor complex during evaluation of symptoms. The jejunum was perfused with $200 \mu \mathrm{g} / \mathrm{ml}$ capsaicin solution and participants recorded any symptoms on the graded questionnaire. When discomfort was reported, perfusion was terminated and the jejunum was perfused with normal saline for at least 10 minutes or until the sensation vanished. Thereafter, this protocol was repeated twice and at each trial the latency of the first sensation threshold and discomfort threshold was determined. In this set of experiments no barostat balloon was used.

\section{Data analysis}

Jejunal tone and phasic activity

Volume and pressure values of the barostat bag were recorded online at a rate of $4 \mathrm{~Hz}$. Jejunal tone was expressed as the mean barostat volume averaged over the last minute of each interval of tone measurement. Jejunal tone was also assessed for the two minutes before and during the period when subjects described discomfort and for a two minute period when sensations had completely vanished. Phasic activity was visually analysed for specific motility patternsthat is, migrating motor complexes, bursts of contractions or clustered contractions, prolonged propagated contractions, and high amplitude contractions at the time when capsaicin induced perceptions. Motility indices $\left(\log _{\mathrm{e}}(\right.$ (sum of amplitudes $\times$ number of waves) +1$)$ ) were calculated for the two minute periods when subjects reported maximal discomfort induced by capsaicin perfusion and thereafter when symptoms had completely vanished ( score $\leqslant 1$ ).

\section{Compliance}

Compliance of the jejunal wall describes the volume response to an imposed distension pressure. The volume needed to reach the preset pressure during distension sequences was recorded.

\section{Perception questionnaire}

For each individual stimulus-response trial, perception thresholds and discomfort thresholds were defined as the smallest balloon pressure or as the duration of capsaicin perfusion, respectively, when perception was reported. Thresholds for perception were determined when the score for perception became $\geqslant 2$ for the first time on one of the graded scales. Discomfort thresholds were defined as being reached when any type of sensation with score 5 or greater (except pain: score 3.5 or greater) was recorded. After capsaicin induced discomfort, the jejunum was perfused with normal saline until the sensation had vanished (score $\leqslant 1)$.

Aggregate scores were calculated by summing the scores for each type of sensation at each distension step and at the time when capsaicin induced discomfort.

Thresholds for perception were expressed as the volume in the bag at the onset of constant sensation. Sensations were considered to be constant when they had lasted at least 30 seconds.

\section{Statistical analysis}

Nominal data are given as mean (SEM) and ordinate data as median (25th and 75th percentiles). For statistical comparisons, parametric (paired Student's $t$ test) and non-parametric (Wilcoxon test, Kruskall-Wallis test) tests were used, 


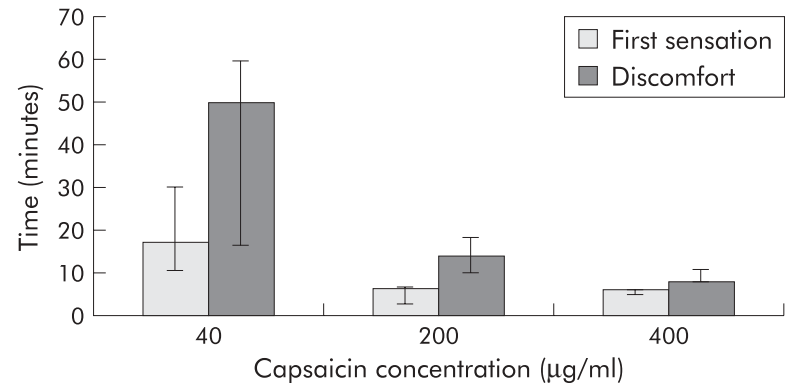

Figure 1 Time until jejunal capsaicin perfusion induced the first sensation and discomfort was concentration dependent. Data are medians (25th-75th percentiles); $\mathrm{n}=19$.

as appropriate. An alpha level of less than 0.05 was considered significant.

\section{RESULTS}

\section{Effects of capsaicin perfusion on perception}

Perfusion of the jejunum with capsaicin induced perception that increased in its intensity as perfusion progressed. The time until the first sensation and discomfort were reported was concentration dependent (fig l) $(\mathrm{p}<0.01)$. All subjects receiving one of the two higher concentrations of capsaicin and seven of 12 subjects receiving $40 \mu \mathrm{g} / \mathrm{ml}$ capsaicin developed discomfort within the 60 minute perfusion period. All but two subjects given the $40 \mu \mathrm{g} / \mathrm{ml}$ concentration developed at least definite but mild sensations during capsaicin perfusion. After the capsaicin perfusion was stopped, perception subsided within 5.0 (2.0) minutes $(40 \mu \mathrm{g} / \mathrm{ml}), 5.2(1.2)$ minutes $(200 \mu \mathrm{g} / \mathrm{ml})$, and $4.4(0.9) \mathrm{min}-$ utes $(400 \mu \mathrm{g} / \mathrm{ml})$, respectively $(\mathrm{p}>0.05)$.

In a second series, five subjects received repeated capsaicin perfusions $(200 \mu \mathrm{g} / \mathrm{ml})$ to evaluate whether repeated applications sensitises or desensitises jejunal nociceptors. Median time to the first sensation decreased from 5.4 minutes (25th75th percentiles 4.2-6.0 minutes) during the first capsaicin perfusion to 1.5 minutes (1.3-2.0) and 1.3 minutes (1.1-1.5) during the second and third capsaicin perfusions, respectively (regression analysis $r^{2}=0.39, p=0.01$ ). Duration of perfusion until discomfort was reported also decreased during the second and third perfusions $\left(p<0.001, r^{2}=0.85\right)$ (fig 2$)$. Time needed until the sensation vanished (perception score

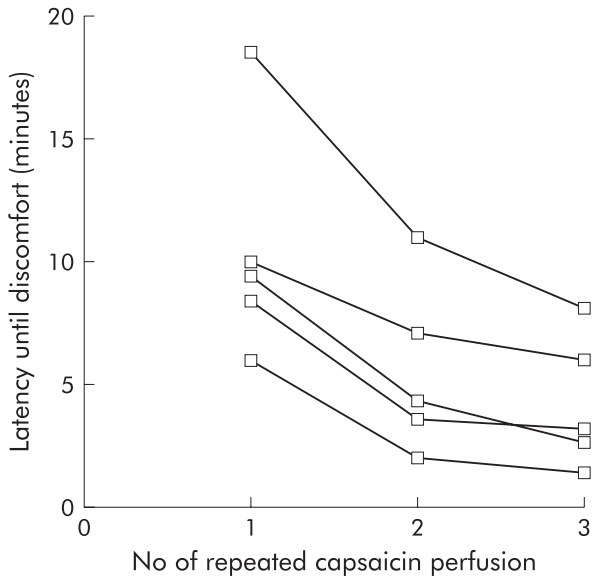

Figure 2 Duration of capsaicin perfusion $(200 \mu \mathrm{g} / \mathrm{ml})$ until discomfort was reported during three consecutive capsaicin perfusion trials. Each curve represents one subject.

$\leqslant 1$ ) was not significantly different after the first (3.3 minutes (25th-75th percentiles 3.1-4.1 minutes)) second (6.2 minutes (4.1-6.5)), and third (6.1 minutes (6.1-7.0)) trials of capsaicin perfusion although there was a tendency for the recovery time to increase after each perfusion trial.

\section{Effect of capsaicin on phasic motility and compliance in the jejunum}

Jejunal tone, expressed as barostat volume at constant operating pressure (12.4 (1.8) $\mathrm{mm} \mathrm{Hg}$ ), was not altered by capsaicin perfusion. In subjects who failed to experience any sensation or discomfort during capsaicin $(40 \mu \mathrm{g} / \mathrm{ml})$ perfusion (group B, $\mathrm{n}=5$ ), barostat volume was 72 (16) ml before capsaicin perfusion started and 62 (19) $\mathrm{ml}$ after 60 minutes of perfusion of $40 \mu \mathrm{g} / \mathrm{ml}$ capsaicin $(\mathrm{p}>0.05)$. In subjects who reported discomfort during capsaicin perfusion $(40-400 \mu \mathrm{g} /$ $\mathrm{ml}$, group A), jejunal tone likewise did not change significantly (57 (4) $\mathrm{ml}$ before the start of perfusion $v 50$ (4) $\mathrm{ml}$ at discomfort; $\mathrm{p}>0.05$ ). Finally, after capsaicin induced discomfort had subsided, barostat volume also did not significantly differ from that measured during discomfort (47 (4) ml) $(\mathrm{p}>0.05)$.
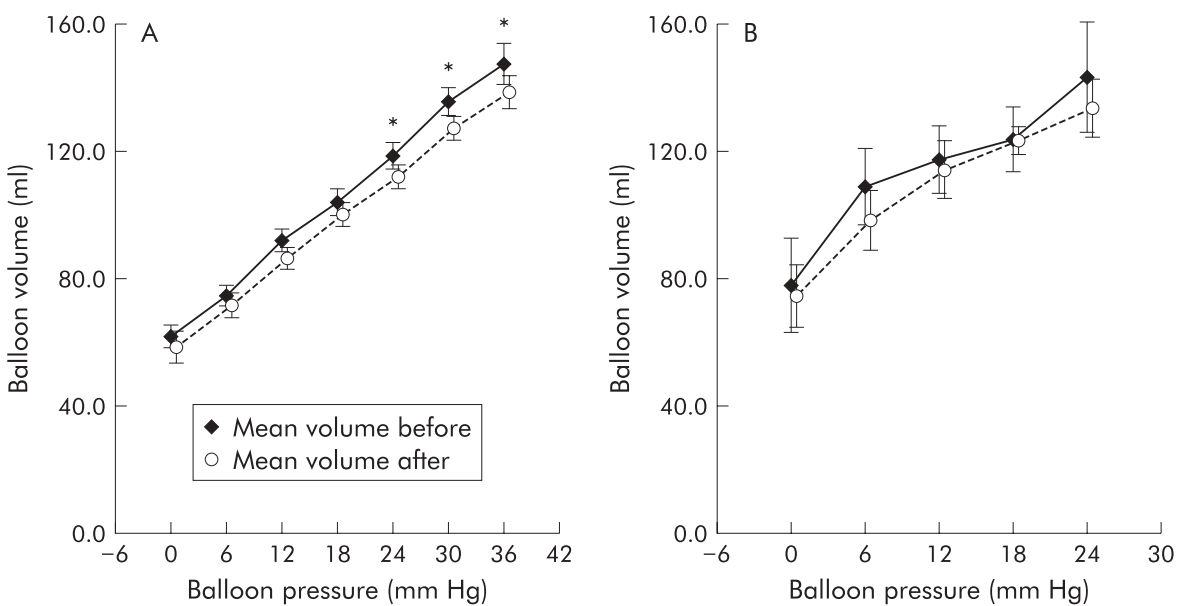

Figure 3 (A) Jejunal compliance determined by balloon inflation before capsaicin perfusion and after capsaicin perfusion was stopped because of development of discomfort ( $\mathrm{n}=19$, group A). At higher distension pressures (24 mm Hg or more), balloon volumes became smaller after capsaicin perfusion. (B) Jejunal compliance determined by balloon inflation before and during capsaicin perfusion (group B). No difference was seen between the two treatments. Data are means (SEM); $n=5$. * $p<0.05$. 

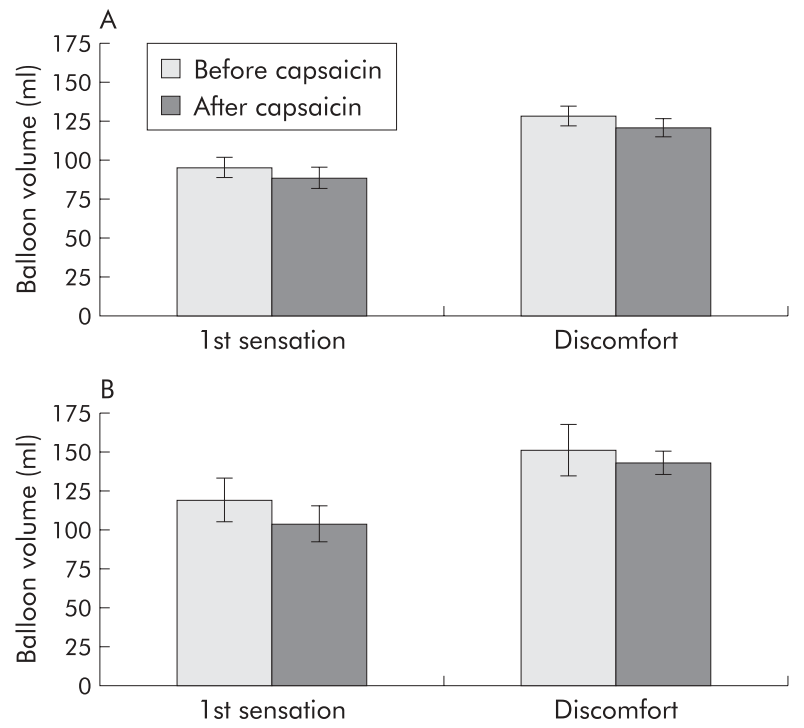

Figure 4 No significant difference in threshold volumes to induce the first sensation and discomfort during distensions performed before capsaicin perfusion and after capsaicin perfusion was stopped because of discomfort (group A) (A), as well as before and during capsaicin perfusion (group B) (B). Data are means (SEM).

Motility indices recorded from port $1(5 \mathrm{~cm}$ proximal to the perfusion site) were significantly lower during the time when perfusion with capsaicin induced discomfort $(6.2(0.8))$ compared with the time when sensations had subsided $(8.2$ (0.7)) $(p=0.02)$. Motility indices at the ports positioned distally to the perfusion site were not significantly different during the period of discomfort (port 2: 4.7 (0.9); port 3: 1.4 (0.6); port $4: 1.8(0.9))$ and the symptom free period thereafter (port 2: 6.1 (0.7); port 3: 2.1 (0.9); port 4: 2.9 (0.9)) ( $p>0.05$ for each). No reproducible patterns of jejunal motility were observed during capsaicin perfusion. The volume in the balloon at higher distending pressures (24 mm Hg and higher) was significantly lower after capsaicin perfusion (distension 2) compared with distension 1 which was performed before capsaicin perfusion (fig 3A). No difference in compliance was seen between distensions before and during capsaicin perfusion (fig 3B).

\section{Perception thresholds during distension}

Thresholds for perception of distension determined after capsaicin perfusion were unchanged compared with thresholds determined before capsaicin perfusion (group A, $\mathrm{n}=19$ ). Volumes of the distending balloon at the time of first perception were 96 (6) $\mathrm{ml}$ and 90 (7) $\mathrm{ml}$ before and after
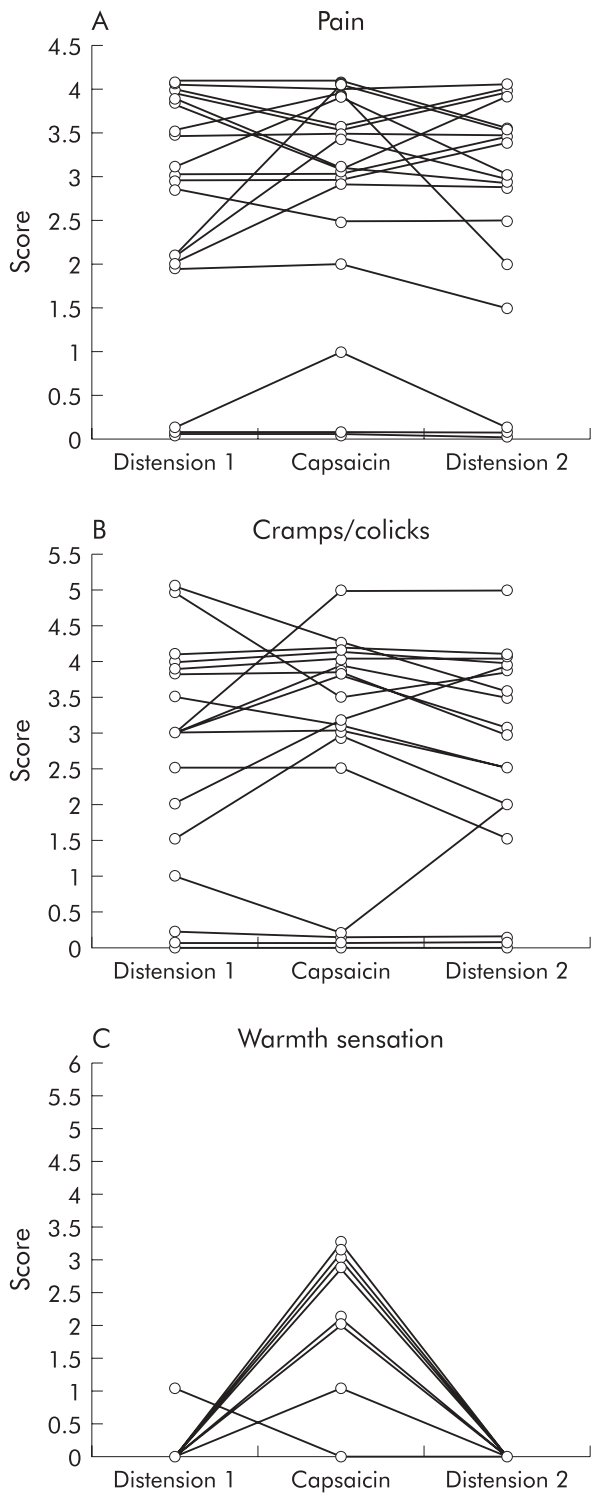

Figure 5 Scores for sensation of pain (A), cramps/colics (B), and warmth $(C)$ reported by each individual subject at the time when distension and capsaicin perfusion, respectively, caused discomfort, and for this reason were stopped. Distension 1, distension before capsaicin perfusion; distension 2, distension after capsaicin perfusion.

capsaicin perfusion $(\mathrm{p}>0.05)$, respectively (fig $4 \mathrm{~A})$. Balloon volumes at the discomfort threshold were 130 (6) $\mathrm{ml}$ before and 123 (6) $\mathrm{ml}$ after capsaicin perfusion ( $\mathrm{p}>0.05$ ) (fig 4A).

Table 1 Median scores (25th-75th percentiles) for sensations at the time of discomfort induced by jejunal distension before and after capsaicin perfusion, and for discomfort induced by capsaicin

\begin{tabular}{llllc}
\hline Sensation & $\begin{array}{l}\text { Distension before capsaicin } \\
\text { perfusion }\end{array}$ & $\begin{array}{l}\text { Capsaicin } \\
\text { perfusion }\end{array}$ & $\begin{array}{l}\text { Distension after capsaicin } \\
\text { perfusion }\end{array}$ & p Value \\
\hline Pressure & $4(3.5-4.5)$ & $3(2-5)$ & $4(3-5)$ & 0.03 \\
Cramps/colic & $3(1.5-4)$ & $3(0-5)$ & $3(2-5)$ & $>0.05$ \\
Stinging & $2(0-3.5)$ & $2(0-4)$ & $2(0-5)$ & $>0.05$ \\
Flutter-like & $0(0-0)$ & $0(0-3)$ & $0(0-2.5)$ & $>0.05$ \\
Warmth & $0(0-0)$ & $0(0-2)$ & $0(0-0)$ & $<0.01$ \\
Vacuum & $0(0-0)$ & $0(0-2.5)$ & $0(0-2.5)$ & $>0.05$ \\
Pain & $3(2-4)$ & $3.5(2-4)$ & $3(2-4)$ & $>0.05$ \\
\hline \multicolumn{7}{l}{ p values denote comparisons of capsaicin with distensions. } \\
\hline
\end{tabular}




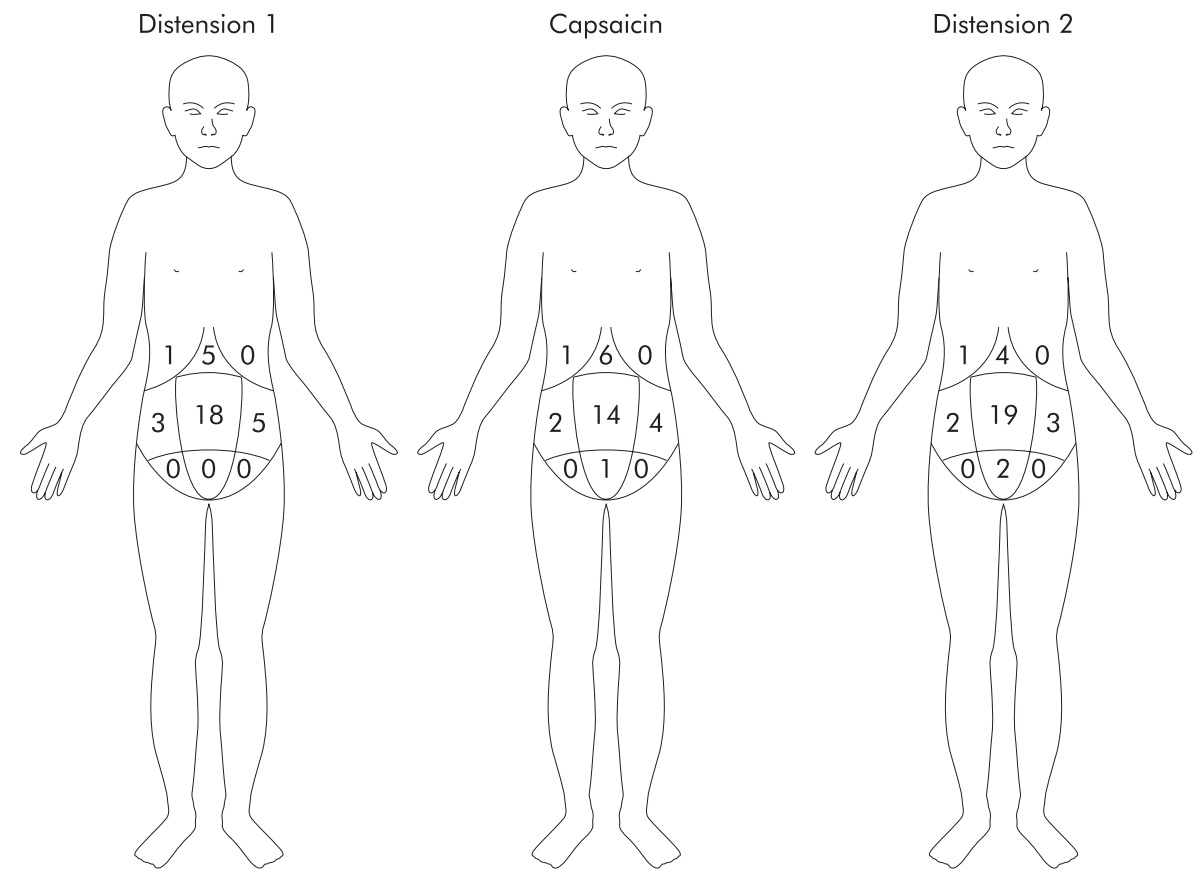

Figure 6 Referral of symptoms to the abdominal surface. Numbers in the abdominal areas denote number of subjects who reported symptoms in the respective area. Most subjects selected more than one area.

Similarly, perception thresholds of distension were not altered by simultaneous capsaicin perfusion (group B, $\mathrm{n}=5$ ). Volumes of the distending balloon at the time of first perception were 119 (14) $\mathrm{ml}$ and 103 (12) $\mathrm{ml}$ before and during capsaicin perfusion, respectively $(\mathrm{p}>0.05)$ (fig 4B). Balloon volumes at the discomfort threshold were 152 (16) $\mathrm{ml}$ before and 143 (6) $\mathrm{ml}$ during capsaicin perfusion $(\mathrm{p}>0.05)$ (fig 4B).

\section{Quality of perception}

The quality of perceptions induced by capsaicin perfusion were mainly described as pressure/fullness, cramps/colic, stinging sensation, and pain. Most of the perception qualities and intensity of capsaicin induced perceptions were comparable with those induced by balloon distension, as shown in fig 5 and table 1 . However, the perception of pressure was less intense during capsaicin $(p=0.03)$. Sensations of flutterness, warmth, and vacuum were reported inconsistently, but warmth was a quality of sensation that was attributed to capsaicin perfusion only. Aggregate perception scores at the time of discomfort were similar during distension l (11.6 (3.3)), capsaicin perfusion (11.8 (3.3)), and distension 2 (11.3 (3.0)) $(\mathrm{p}>0.05)$. At the time of discomfort, symptoms were mainly referred to the midline (periumbilical region) both during distension and capsaicin perfusion of the jejunum (fig 6).

\section{DISCUSSION}

In a previous study, we have demonstrated that capsaicin, the pungent ingredient of chili pepper, induces pain when infused into the jejunum, ${ }^{12}$ although the study design did not allow us to infer whether capsaicin caused pain directly by stimulating nociceptive afferents in the intestinal mucosa or indirectly by stimulating motility. The results of the current study demonstrate that perception induced by capsaicin has qualitative features which are similar but not identical to perception induced by mechanical distension. Capsaicin induced nociception was not associated with specific motor patterns or alterations in tonic motility. Repeated capsaicin perfusions result in sensitisation of nociceptors but capsaicin did not alter perception of mechanical distension of the jejunum. Thus our results suggest that capsaicin induces nociception independently of mechanoreceptors via a specific capsaicin sensitive chemoreceptive pathway.

Our previous observations, which showed that cramps are a predominant feature of capsaicin induced jejunal perception, raised the hypothesis that symptoms during capsaicin perfusion might be secondary to stimulation of intestinal motility. Chilli and Tabasco, both pungent varieties of chilli pepper that contain capsaicin but also other compounds in the group of capsacinoids, have been shown to accelerate intestinal transit. ${ }^{23}{ }^{24}$ Thus one aim of the current study was to evaluate whether an increase in jejunal tonic or phasic motor activity could explain the pain evoked by jejunal capsaicin perfusion. However, tone was not altered and we did not see any consistent motor pattern that might indicate stimulation of jejunal motility by pure capsaicin. In contrast, at the time when discomfort occurred during capsaicin perfusion, phasic motor activity was significantly reduced proximal to the perfusion site. Orad reflex inhibition of phasic motor activity has been demonstrated as a response to mechanical distension. ${ }^{3}$ We also consider this effect of capsaicin perfusion as an orad reflex inhibition rather than a direct effect of capsaicin on motor activity. However, in vitro, capsaicin inhibits both longitudinal and circular muscle activity, ${ }^{15}{ }^{16}$ and thus a direct effect of capsaicin on muscle activity cannot be excluded. There was a slight but significant decrease in jejunal compliance that we detected only when symptoms had disappeared after capsaicin had caused painful sensations. This might also have been caused by local reflexes, although a direct effect of capsaicin on jejunal compliance cannot be excluded in view of in vitro studies. ${ }^{15} 16$ Based on studies by others which have shown good reproducibility of intestinal distension trials, ${ }^{5}$ we consider an order effect of repeated distensions on compliance or perception of distension to be unlikely. 
The intestinal mucosa with its immediate contact with intestinal contents not only provides a vast area for the absorption of nutrients but also acts as a sensory organ. ${ }^{25}$ Investigation of visceral sensitivity employs test stimuli that activate afferent pathways and induce perception. In the human, balloon distension and electrical stimulation have been used for nearly a century in this respect. ${ }^{26}$ It has been shown that perception induced by distension and transmucosal electrical stimulation are stimulus related and induce symptoms that are similar to those in patients with functional bowel disorders. Chemoreceptors have long been known to be important regulators of gastrointestinal function and to mediate local reflexes that control motility, secretion, and vascular perfusion. They are involved in viscero-visceral reflexes and also convey information to the central nervous system. ${ }^{25}$ Chemoreceptors are also known to have the potential to alter sensations arising from the viscera ${ }^{28}$ and are involved in nociception due to mediator release induced by inflammation and ischaemia. The potential role of chemoreceptors in gastrointestinal diseases which lack these organic alterations, such as in functional bowel disorders, has so far been ignored, partly due to lack of a pathophysiological human in vivo model. Our model of capsaicin induced nociception provides evidence that intraluminal contents can modulate perception and cause discomfort or pain in a normal appearing gut, and thus provides a model for the study of functional bowel disorders.

In our study, we have shown that symptoms due to capsaicin are similar to those resulting from mechanical distension of the jejunum. Nevertheless, capsaicin neither sensitised nor desensitised mechanoreceptors, as perception thresholds for distension were similar before, during, and after capsaicin perfusion. On the other hand, repeated capsaicin application had a pronounced sensitising effect on chemonociceptors, as shown by a pronounced decrease in the time until sensation thresholds were reached. This feature clearly differentiates capsaicin induced sensations from distension induced sensations. Another important argument in this line is provided by the observation that the sensation of warmth occurred only with capsaicin perfusion but not with distension. Our findings therefore strongly suggest that capsaicin induced sensations were evoked by stimulation of a specific receptor. A likely candidate would be vanilloid receptor type 1 which is a polymodal receptor that is not only activated by capsaicin but also by noxious heat, acid, and ethanol, and whose activation, independent of the stimulus, gives rise to a "hot" perception quality. ${ }^{17} 1829$

One methodological issue has to be raised however. The possibility exists that in the experiments utilising repeated capsaicin perfusion, the 10 minute washout phases between capsaicin perfusions were not sufficiently long to clear the investigated region of capsaicin. Although we cannot exclude this possibility, we believe that the decrease in latency until first sensation and discomfort from the first to the second and again from the second to the third infusion periods makes this unlikely. However, further studies employing longer washout phases or examining other agents known to stimulate vanilloid type 1 receptors are needed to clarify capsaicin induced sensitisation of the jejunum.

Neither preceding nor simultaneous capsaicin perfusion of the jejunum resulted in sensitisation to mechanical distension in our hands. Others have shown that hyperalgesia to distension occurs after capsaicin bolus application into an ileostoma. ${ }^{13}$ This discrepancy may be due to important differences in methodology, bowel region, and the condition under study. It should in particular be considered that, in the postoperative gut, capsaicin may stimulate neural afferents as well as mast cells in a vanilloid receptor mediated fashion, ${ }^{30}$ the inflammatory mediators released from these cells leading to mechanoreceptor sensitisation.

Our data do not allow us to conclude that capsaicin induced nociception was mediated by mucosal receptors, by receptors in deeper parts of the gut wall, or by stimulation of the gut endocrine system. However, the short time period needed for pain to disappear when capsaicin perfusion was stopped and luminal concentrations of capsaicin were diluted with normal saline argues for an action of capsaicin on mucosal nerve fibres. Vanilloid type 1 receptors are expressed by many dorsal root ganglion cells in rats ${ }^{17} 3132$ and humans. ${ }^{33}$ Some of these neurones supply the gastrointestinal tract, and immunohistochemistry has shown that vanilloid type 1 receptor positive nerve fibres of extrinsic origin project to the musculature, submucosa, and mucosa of the human colon. ${ }^{33}$ Interestingly, the number of vanilloid type 1 receptors in the colonic submucosa is significantly enhanced in patients with painful inflammatory bowel disease. ${ }^{33}$ From the available evidence it would appear that in the human gut, vanilloid type 1 receptors are confined to spinal afferent nerve fibres, ${ }^{33}$ and that it is stimulation of these afferents whereby capsaicin gives rise to pain, as shown in this and other studies. $^{12} 3435$

We have assessed sensation in two different ways. The aggregate perception score assumes that different sensations occur simultaneously, and provides information on the subjective intensity of overall perception. In contrast, perception thresholds assume that the different sensations develop gradually one after the other as perfusion continues. Both during distension as well as during capsaicin infusion, we have observed simultaneous occurrence of several different qualities of perception which gradually increased as the intensity of stimulation was increased. We have found qualitatively similar sensations during capsaicin perfusion compared with distension, although the feeling of pressure tended to be reported more often during distension (not significant after Bonferroni's correction for multiple comparisons) whereas the feeling of warmth, a less frequent sensation, was nearly exclusively reported during capsaicin perfusion. Interestingly, clinical practice teaches that the sensation of warmth may also be reported by some patients with functional bowel disorders, although this is rarely the predominant symptom.

Symptoms in functional bowel disorders are related to abnormal sensation. ${ }^{36}$ Afferent nerve pathways play a role in lowered perception thresholds and visceral hypersensitivity, ${ }^{1}$ but the currently available methods, mainly balloon distension, do not detect abnormal perception in all patients. ${ }^{37} 38$ Evaluation by a combination of different stimulation techniques to define the gastrointestinal sensory dysfunction in different subsets of patients with unexplained abdominal symptoms might improve the diagnostic workup and, in the future, allow for a differential therapy depending on the predominant sensory dysfunction.

While the type of the abdominal symptoms, which patients with functional bowel disorders experience during mechanical distension and electrical stimulation, are similar to sensations in healthy subjects, mechanical but also electrical stimuli are more diffusely perceived over the abdomen by patients compared with healthy controls. ${ }^{3}$ This suggests that patients with functional bowel disorders may have sensory disturbances at the receptor level and disturbances of spinal processing of sensory information. In our study, referral of symptoms was similar during distension induced discomfort and capsaicin induced discomfort, suggesting the same spinal processing of both stimulation modalities.

It has recently been reported that a five week trial of patients with functional dyspepsia with red pepper initially evokes epigastric pain or discomfort. ${ }^{39}{ }^{40}$ After a few days of 
administration, however, pain and discomfort diminished and dyspepsia symptoms improved. The therapeutic effect was explained by a decrease in afferent nerve fibre activity after chronic repeated application of capsaicin. ${ }^{14}$ This interpretation is consistent with the pharmacology of vanilloid type 1 receptor bearing sensory neurones which acutely are stimulated by capsaicin but on long term exposure to the drug are defunctionalised and thereby become insensitive to noxious stimuli. ${ }^{13} 39$

Genetic deletion of vanilloid type 1 receptors in mice leads to impaired chemonociception and impaired inflammatory thermal hyperalgesia. ${ }^{41}{ }^{42}$ In view of the integrative role of vanilloid type 1 receptors in the sensing of noxious stimuli, it may be envisaged that this receptor is a chemosensor for endogenous mediators that may play a role in symptoms of functional bowel disorders. If so, intestinal chemonociception evoked by capsaicin may represent a pathophysiological model for functional bowel disorders. This conjecture raises the possibility that blockade of vanilloid type 1 receptors may be a novel therapeutic approach for treatment of some patients suffering from functional bowel disorders. Indeed, capsaicin analogues that lack an excitatory effect on vanilloid type 1 receptors but are able to block the function of vanilloid type 1 receptor bearing nociceptors ${ }^{43}$ as well as competitive vanilloid type 1 receptor antagonists ${ }^{44}$ are currently under development as novel antinociceptive agents.

In summary, our study provides a new model to examine intestinal nociception evoked by luminal stimuli that involves specific intestinal chemoreceptors but does not depend on mechanoreceptors. It also offers a novel pathophysiological approach to the study of functional bowel disorders. However, hypersensitivity of chemical nociceptors in patients with functional bowel disorders needs to be established. Specific antagonists of vanilloid type 1 receptors might be of therapeutic value in patients who demonstrate hypersensitivity of chemical nociceptors.

\section{ACKNOWLEDGEMENTS}

Supported by the research grant P15036 from the Austrian Science Fund (FWF, Fonds zur Förderung der wissenschaftlichen Forschung) to $\mathrm{JH}$.

\section{Authors' affiliations}

B Schmidt, J Hammer, Abteilung für Gastroenterologie und Hepatologie, University of Vienna, Vienna, Austria

P Holzer, Institut für Experimentelle und Klinische Pharmakologie, University of Graz, Graz, Austria

H F Hammer, Medizinische Universitätsklinik Graz, Graz, Austria

\section{REFERENCES}

1 Mayer EA, Gebhart GF. Basic and clinical aspects of visceral hyperalgesia. Gastroenterology 1994;107:271-93.

2 Hammer HF, Krejs GJ. Acute abdomen. In: Bianchi Porro G, Cremer M, Krejs G, et al, eds. Gastroenterology and hepatology (Clinical Medicine Series). London: McGraw-Hill, 1999:49-53.

3 Accarino AM, Azpiroz F, Malagelada J-R. Selective dysfunction of mechanosensitiv intestinal afferents in irritable bowel syndrome. Gastroenterology 1995;108:636-43.

4 Accarino AM, Azpiroz F, Malagelada J-R. Symptomatic responses to stimulation of sensory pathways in the jejunum. Am J Physiol 1992;263:G673-7.

5 Accarino AM, Azpiroz F, Malagelada J-R. Gut perception in humans is modulated by interacting gut stimuli. Am J Physiol Gastrointest Liver Physiol 2002;282:G220-5.

6 Holtmann G, Goebell H, Jockenhoevel F, et al. Altered vagal and intestinal mechanosensory function in chronic unexplained dyspepsia. Gut 1998:42:501-6.

7 Coffin B, Azpiroz F, Guarner F, et al. Selective gastric hypersensitivity and reflex hyproreactivity in functional dyspepsia. Gastroenterology 1994; 107:1345-51.

8 Greydanus MP, Vassallo M, Camilleri M, et al. Neurohormonal factors in functional dyspepsia: insights on pathophysiological mechanisms. 1991;100:1311-18.
9 Moriarty KJ, Dawson AM. Functional abdominal pain: further evidence that the whole gut is affected. BMJ 1992;284:1670-2.

10 George AA, Tsuchiyose M, Dooley CP. Sensitivity of the gastric mucosa to acid and duodenal contents in patients with nonulcer dyspepsia. Gastroenterology 1991;101:3-6.

11 Grundy D, Scratchered T. Sensory afferents from the gastrointestinal tract. In: Schultz SG, Wodd JD, Rauner BR, eds. Handbook of physiology, Section 6. The gastrointestinal system. Bethesda: American Physiology Society, 1989:593-620.

12 Hammer J, Hammer HF, Eherer AJ, et al. Intraluminal capsaicin does not affect fluid and electrolyte absorption in the human jejunum but causes pain. Gut 1998;43:252-5.

13 Drewes AM, Schipper K-P, Dimcerski G, et al. Gut pain and hyperalgesia induced by capsaicin: a human experimental model. Pain 2003:104:333-41.

14 Holzer P. Capsaicin: cellular targets, mechanisms of action, and selectivity for thin sensory neurons. Pharmacol Rev 1991;43:143-201.

15 Maggi CA, Patacchini R, Santicioli $P$, et al. Specific motor effects of capsaicin on human jejunum. Eur J Pharmacol 1988;149:393-5.

16 Maggi CA, Giuliani S, Santicioli P, et al. Direct evidence for the involvement of vasoactive intestinal polypeptide in the motor response of the human isolated ileum to capsaicin. Eur J Pharmacol 1990;185: 169-78.

17 Caterina MJ, Schumacher MA, Tominaga $M$, et al. The capsaicin receptor: a heat-activated ion channel in the pain pathway. Nature 1997;389:816-24.

18 Tominaga M, Caterina MJ, Malmberg AB, et al. The cloned capsaicin receptor integrates multiple pain-producing stimuli. Neuron 1998;21:531-43

19 Szallasi A, Di Marzo V. New perspectives on enigmatic vanilloid receptors. Trends Neurosci 2000;23:491-7.

20 Zygmunt PM, Petersson J, Andersson DA, et al. Vanilloid receptors on sensory nerves mediate the vasodilator action of anandamide. Nature 1999;400:452-7.

21 Hwang SW, Cho H, Kwak J, et al. Direct activation of capsaicin receptors by products of lipoxygenases: endogenous capsaicin-like substances. Proc Natl Acad Sci U S A 2000;97:6155-60.

22 Steadman CJ, Phillips SF, Camilleri M, et al. Variation of muscle tone in the human colon. Gastroenterology 1991;101:373-81.

23 Horowitz M, Wishart J, Maddox A, et al. The effect of chilli on gastrointestinal transit. J Gastroenterol Hepatol 1992;7:52-6.

24 Gonzalez R, Dunkel R, Koletzko B, et al. Effect of capsaicin-containing red pepper sauce suspension on upper gastrointestinal motility in healthy volunteers. Dig Dis Sci 1998;43:1165-71.

25 Furness JB, Kunze WAA, Clerc N. Nutrient tasting and signaling mechanisms in the gut II. The intestine as a sensory organ: neural, endocrine, and immune responses, Am J Physiol 1999;277:G922-8.

26 Hertz AF. The sensibility of the alimentary tract in health and disease. Lancet $1911 ; 1: 1051-6$.

27 Bueno L, Fioramonti J, Delvaux $M$, et al. Mediators and pharmacology of visceral sensitivity: from basic to clinical investigations. Gastroenterology 1997;112:1714-43

28 Gebhart GF. Pathobiology of visceral pain: molecular mechanisms and therapeutic implications. IV. Visceral afferent contributions to the pathobiology of visceral pain. Am J Physiol Gastrointest Liver Physiol 2000;278:G834-8.

29 Trevisani M, Smart D, Gunthorpe MJ, et al. Ethanol elicits and potentiates nociceptor response via the vanilloid receptor-1. Nat Neurosci 2002;5:546-51.

30 Biro T, Maurer M, Modarres S, et al. Characterization of functional vanilloid receptors expressed by mast cells. Blood 1998;91:1332-40.

31 Guo A, Vulchanova L, Wang J, et al. Immunocytochemical localization of the vanilloid receptor 1 (VR1): relationship to neuropeptides, the P2X3 purinoceptor and IB4 binding sites. Eur J Neurosci 1999;11:946-58.

32 Michael GJ, Priestley JV. Differential expression of the mRNA for the vanilloid receptor subtype 1 in cells of the adult rat dorsal root and nodose ganglia and its downregulation by axotomy. J Neurosci 1999;19:1844-54.

33 Yiangou Y, Facer P, Dyer NH, et al. Vanilloid receptor 1 immunoreactivity in inflamed human bowel. Lancet 2001;357:1338-9.

34 Gonzalez R, Dunkel R, Koletzko B, et al. Effect of capsaicin-containing red pepper sauce suspension on upper gastrointestinal motility in healthy volunteers. Dig Dis Sci 1998;43:1165-71.

35 Rodriguez-Stanley S, Collings KL, Robinson M, et al. The effects of capsaicin on reflux, gastric emptying and dyspepsia. Aliment Pharmacol Ther 2000; 14:129-34

36 Azpiroz F. Hypersensitivity in functional gastrointestinal disorders. Gut 2002;51(suppl 1):i25-8.

37 Camilleri $M$. Testing the sensitivity hypothesis in practice: tools and methods, assumptions and piffalls. Gut 2002;51(suppl I):i34-40.

38 Delvaux M. Role of visceral sensitivity in the pathophysiology of irritable bowel syndrome. Gut 2002;51(supp I)::67-71.

39 Bortolotti M, Coccia G, Grossi G, et al. The treatment of functional dyspepsia with red pepper. Aliment Pharmacol Ther 2002;16:1075-82.

40 Bortolotti M, Coccia G, Grossi G. Red pepper and functional dyspepsia. N Engl J Med 2002;346:947-8.

41 Caterina MJ, Leffler A, Malmberg AB, et al. Impaired nociception and pain sensation in mice lacking the capsaicin receptor. Science 2000;288:306-13.

42 Davis JB, Gray J, Gunthorpe MJ, et al. Vanilloid receptor-1 is essential for inflammatory thermal hyperalgesia. Nature 2000;405:183-7.

43 Urban L, Campbell EA, Panesar M, et al. In vivo pharmacology of SDZ 249665, a novel, non-pungent capsaicin analogue. Pain 2000;89:65-74.

44 Wang Y, Szabo T, Welter JD, et al. High affinity antagonists of the vanilloid receptor. Mol Pharmacol 2002;62:947-56. 